\title{
Design Pattern for the Adaptive Scheduling of Real-Time Tasks with Multiple Versions in RTSJ
}

\author{
Rodrigo Gonçalves, Rômulo Silva de Oliveira, Carlos Montez \\ LCMI - Depto. de Automação e Sistemas - Univ. Fed. de Santa Catarina \\ (rpg,romulo,montez)@das.ufsc.br
}

\begin{abstract}
This paper presents a design pattern of an adaptive scheduling based on the management of the tasks execution time, achieved through multiple versions of the tasks, applied to the Real-Time Specification for Java. A structure of classes is used to facilitate the development of tasks, while allowing the independence of the application code from the code responsible for the adaptive control. The design pattern is described through UML diagrams and an example implementation is presented.
\end{abstract}

\section{Introduction}

The Real-Time Specification for Java (RTSJ) is an extension of the standard Java platform that is able to attend the restrictions imposed by real-time systems, such as predictability and determinism $[1,2,3]$. The RTSJ adds to the Java standard the following characteristics: it adds real-time threads, it allows the execution of code without the interference of the garbage collector, it also allows the control of the localization of objects and the access to memory physical addresses; it implements a manager of asynchronous events and a mechanism for asynchronous transference of control between threads $[1,2,3]$.

An area where the RTSJ will be very important in the next years is the network control. In this type of application, the control of industrial processes is made through a network. Sensors, controllers and actuators are spread by diverse processors in a distributed environment. The control of the industrial process presents real-time requirements and it is affected by the delays in the communication network and the involved processors.

A way to deal with the inherent difficulties to the fulfillment of real-time requirements in distributed environments is the adaptive scheduling. In dynamic environments that are managed by adaptive scheduling, the conditions in which the tasks are executed are defined during the execution of the application itself. One of the forms described in literature to provide such adaptability is to allow tasks to have many versions. In the case of network control, there can be two versions of the controller, a fast one but of inferior quality, and another one with maximum quality but increased execution time. The execution time of the task can be dynamically modified in run-time.

The objective of this work is to elaborate a design pattern defining a class structure capable to encapsulate the code associated with the multiple versions of a task (code that implements different versions of an algorithm). It will facilitate the implementation of new tasks, at the same time it makes the application algorithm independent of the adaptation mechanism. Thus, a programmer can specialize itself only in the development of the application, not having knowledge of how the adaptive control was implemented internally. Another programmer will be responsible for the control classes and the definition of the scheduling algorithm to be used. The main goal is to facilitate the development of real-time applications that apply adaptive scheduling, making possible the reuse of code and allowing the separation of the scheduling algorithm from the application tasks. The use of design patterns represents reduction of costs and greater agility in the implementation.

The next sections present the design pattern considered for the adaptive scheduling based on the management of the tasks execution time. UML diagrams show the structure of the solution and its relationships, as well as the events waited during the execution of the system. Experiences had been carried through to evaluate the behavior of this solution and the result is shown. 


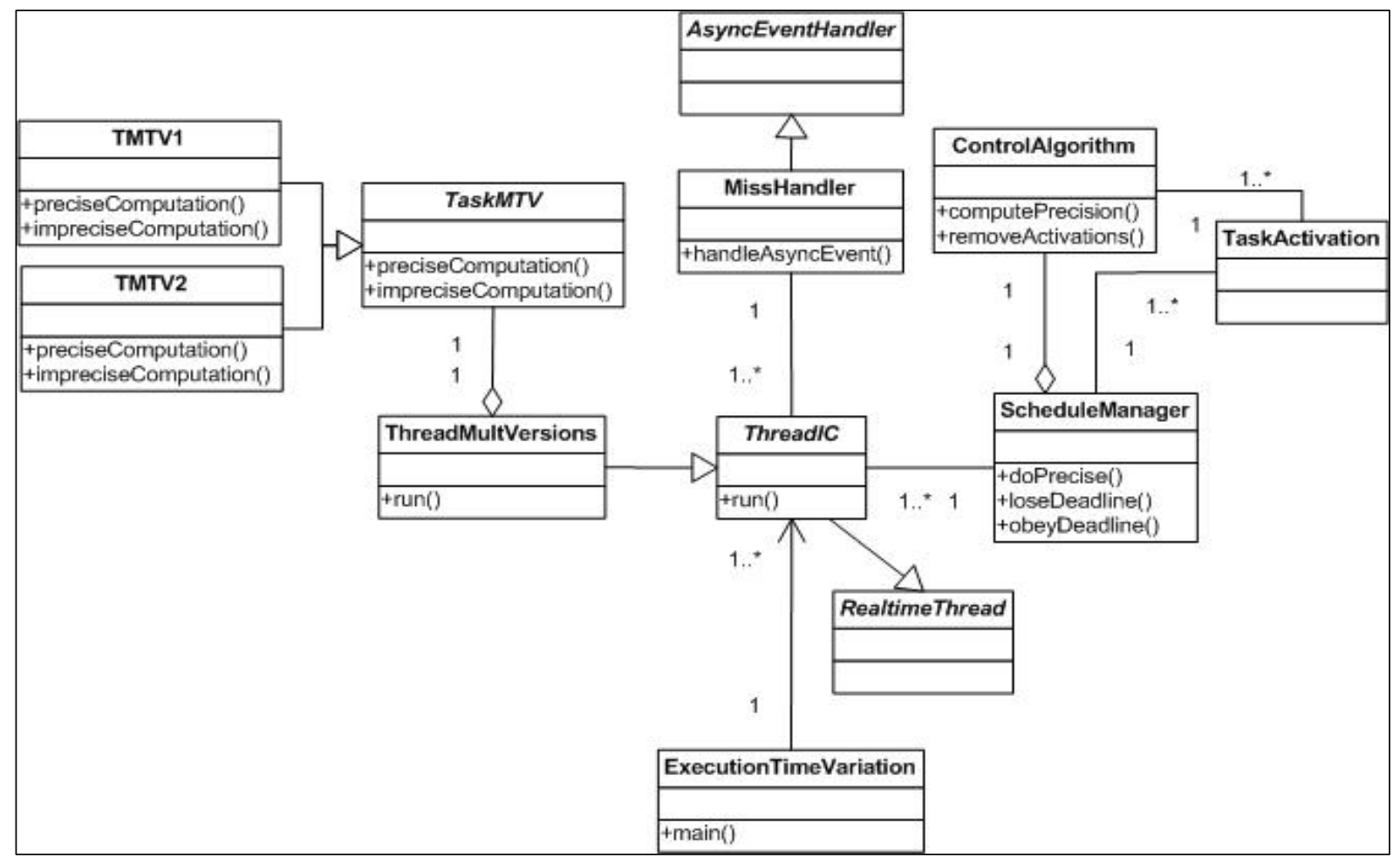

Figure 1. UML class diagram for the proposed solution.

\section{Adaptation of the Execution Time in RTSJ Based on Multiple Versions}

The solution proposed for the management of the execution time is shown by the UML class diagram of figure 1. The method main() is implemented in the class ExecutionTimeVariation, where it initiates the execution of the application by creating threads with multiple versions. The initialization happens according the sequence of events shown in figure 2. Following, figure 3 shows a small part of code of this class with the creation of imprecise threads.

The solution proposed utilizes periodic real-time threads. However, the proposal presented in this paper, and described by figure 1, does not hinder the utilization of others typed of tasks, such as aperiodic or sporadic. We just opted for periodic tasks since them facilitate the observation and analysis of the adaptation of the tasks.

Figure 2 shows the events of the instantiation of an imprecise real-time task. The mechanism of multiple versions is implemented by the ThreadMultVersions class, that is a specialization of the ThreadIC class (it initializes the parameters of threads with multiple versions), and its objects are instantiated by the ExecutionTimeVariation. The ThreadMultVersions class supplies the necessary structure for the execution of the real-time task, by consulting the scheduling on which version of the task it will be carried through, the precise or the imprecise version.

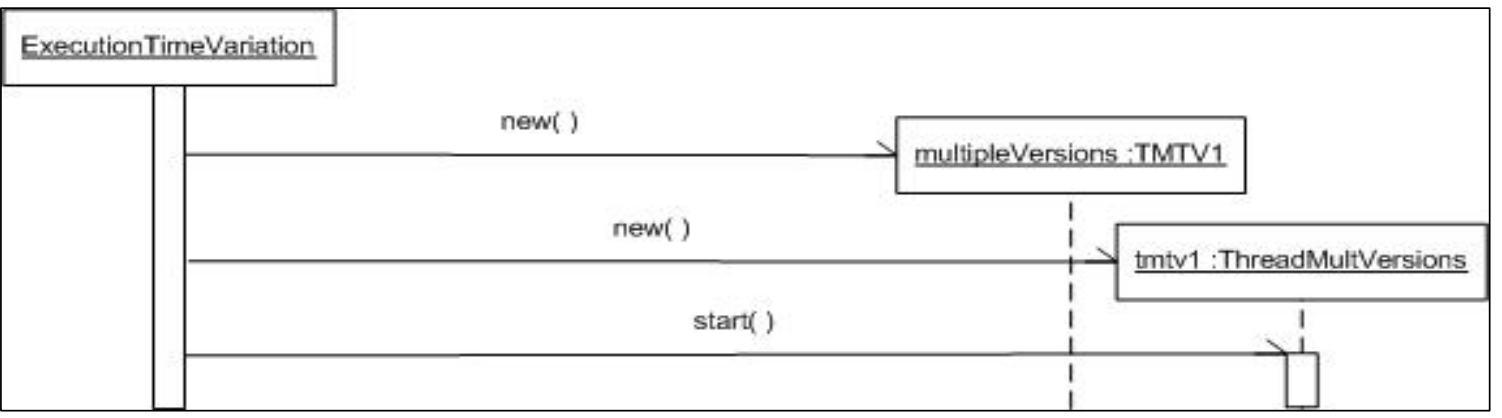

Figure 2. UML sequence diagram of the creation of a thread with multiple versions. 


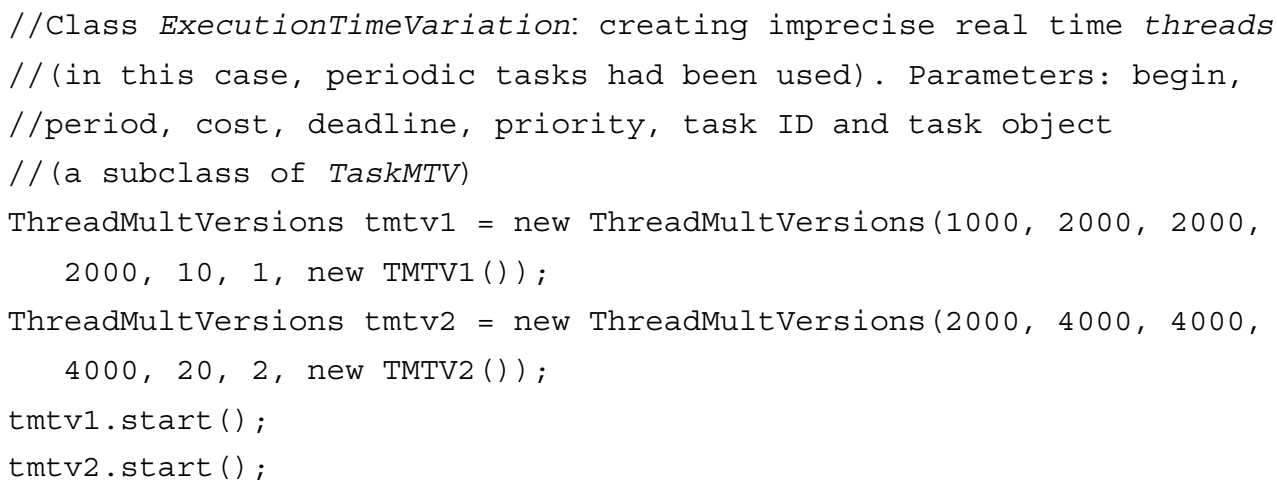

Figure 3. Part of the code of class ExecutionTimeVariation.

Figure 4 shows the constructor of the class ThreadIC, which inherits properties from the class RealtimeThread. Its utility is to simplify the programming of the imprecise tasks, since they share the same code implemented in this class, whose function is to construct a real-time thread with the supplied parameters. Figure 5 shows the constructor of the class ThreadMultVersions that calls the constructor of the superclass and associates the imprecise task to be executed by the thread.

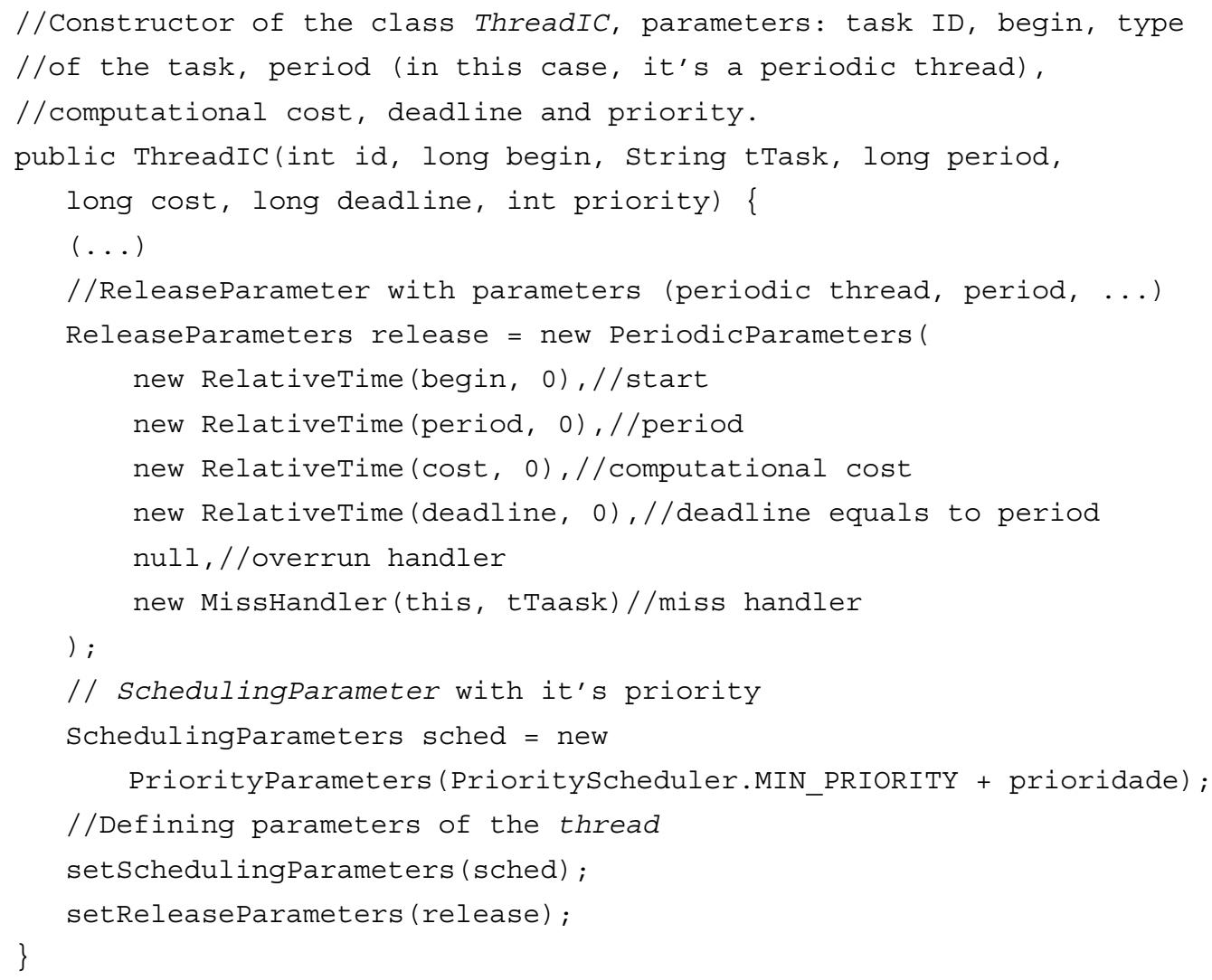

Figure 4. Code for the constructor of class ThreadIC. 


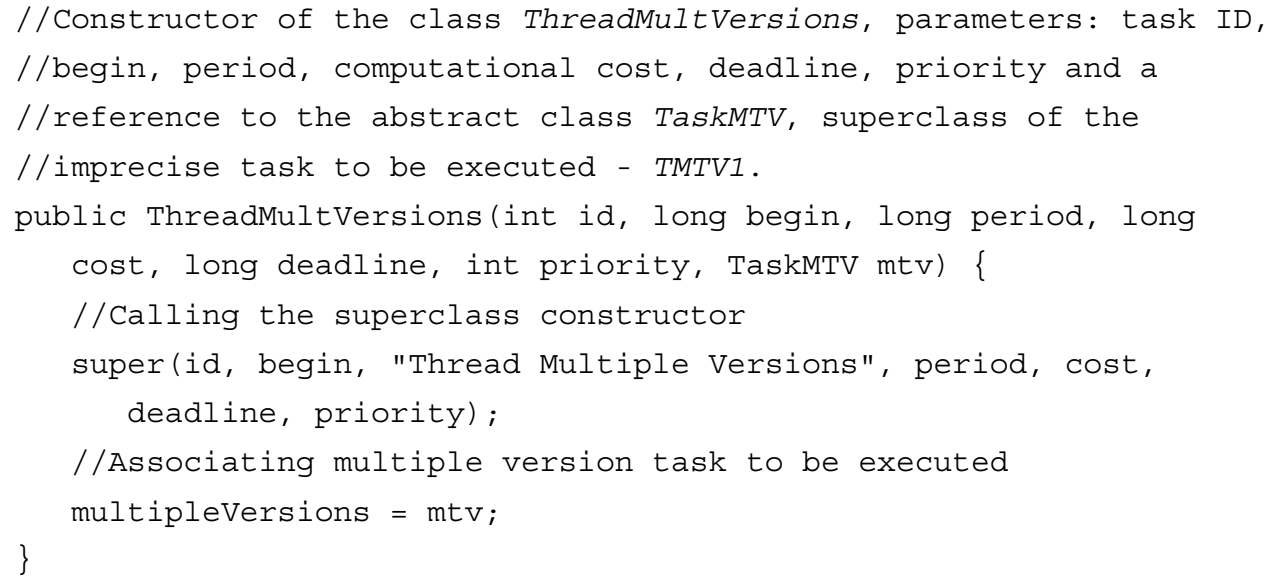

Figure 5. Part of the constructor for class ThreadMultVersions.

The constructor of ThreadIC (figure 4) declares the release parameter (ReleaseParameters) of the thread as PeriodicParameters, which defines the periodic characteristic of the task. The one before last parameter used in the declaration of the object release is called the overrun handler (OverrunHandler). It is executed when the JVM detects that one thread spent more time in the computation than the value defined as its cost.
However, this function is not implemented by the virtual machine used in the experiences. The last parameter is the MissHandler, it answers to an event generated by the JVM when one thread does not finish its execution until the limit defined as its deadline. One of the parameters of this handler is a reference to the thread that caused the event.

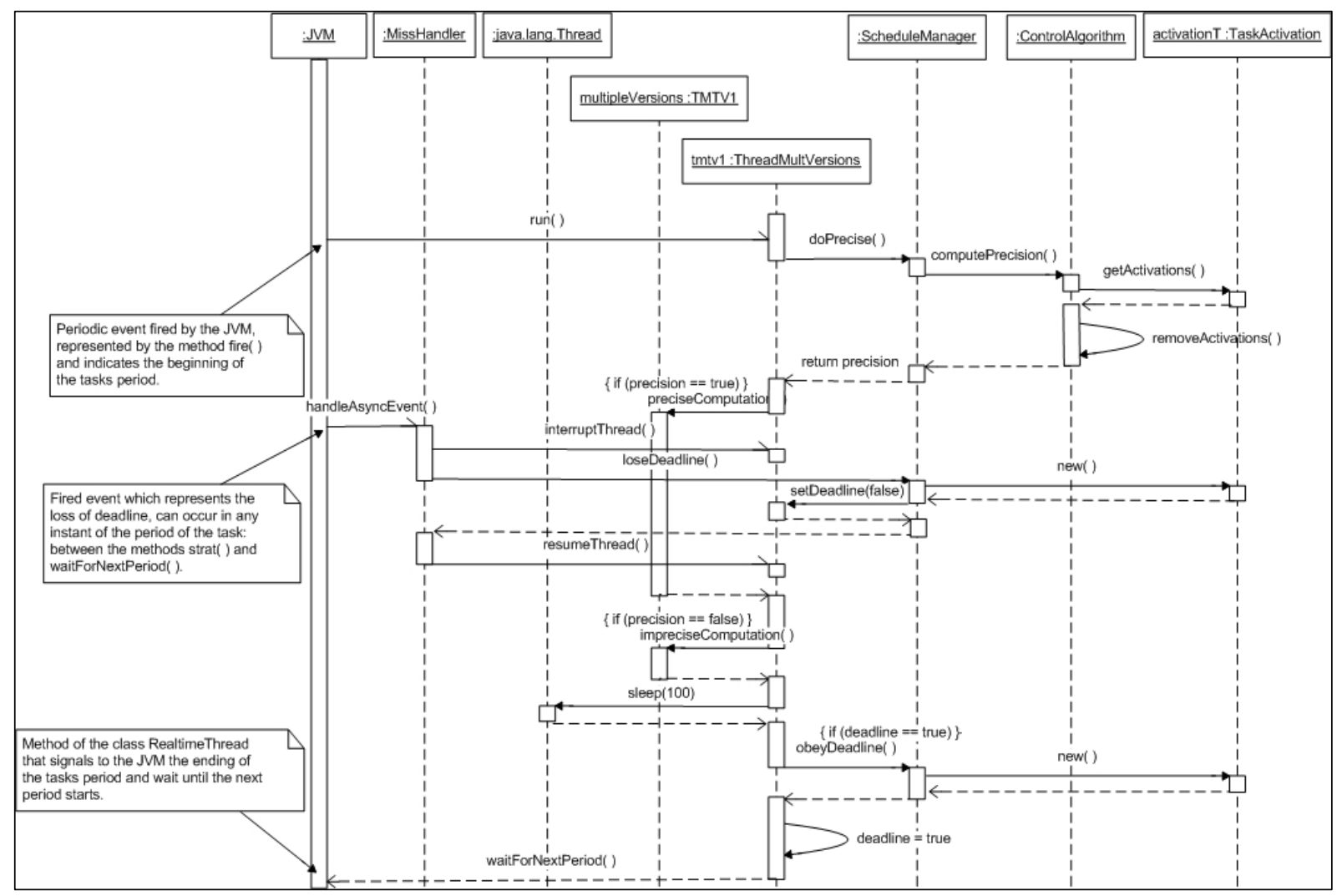

Figure 6. UML sequence diagram for ThreadMultVersions. 


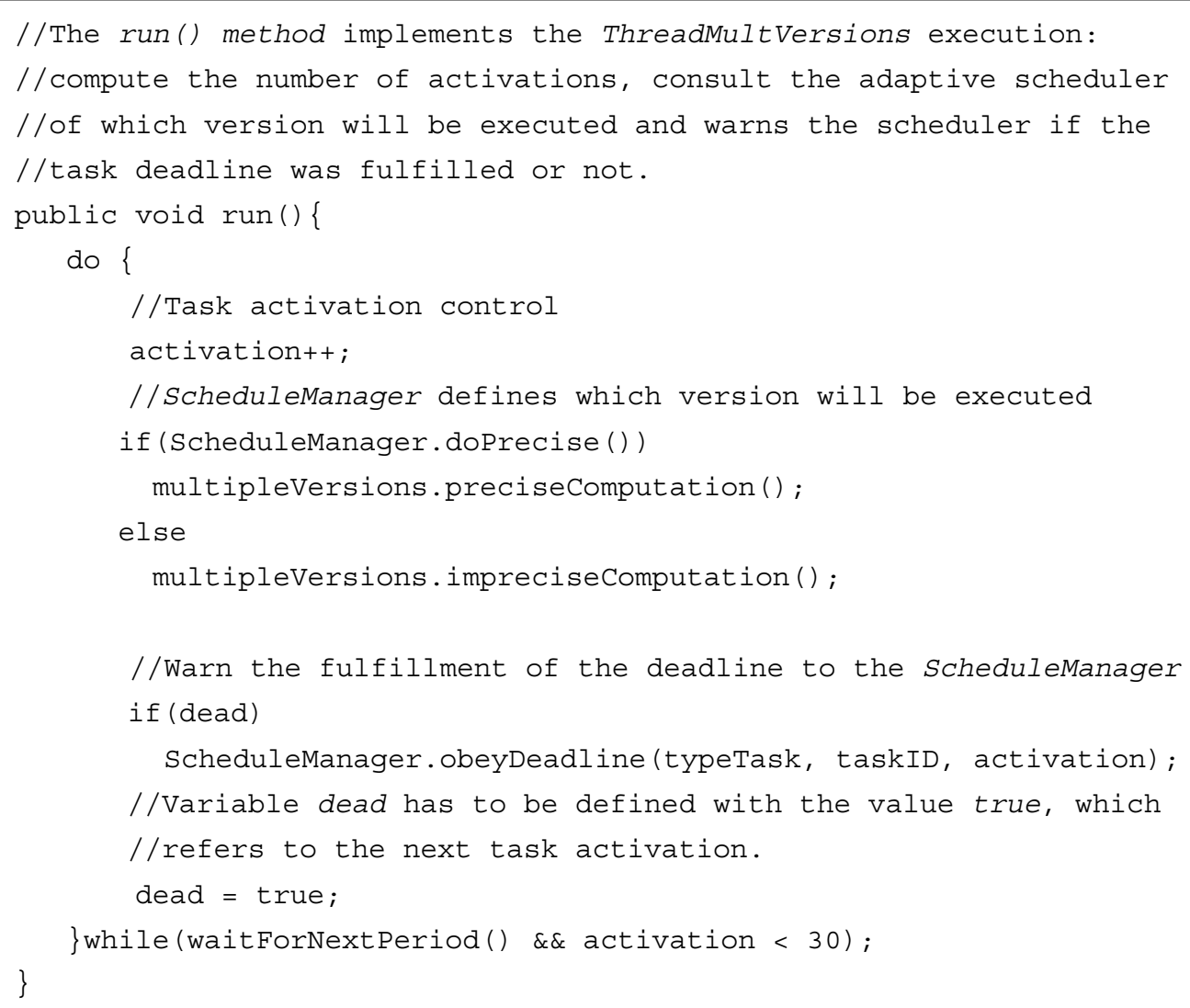

Figure 7. Code for method run() of ThreadMultVersions.

The constructor of the class ThreadMultVersions (figure 5) receives as last parameter a reference to the task to be executed, an object of class TMTV1, and associates this task to the thread just created. This class (TMTV1) is derived from the abstract class TaskMTV.

Figure 6 represents an UML sequence diagram for a period of ThreadMultVersions. It should be noticed that the algorithm initiates consulting to the ScheduleManager on which precision level should be carried through by the task, that then executes the precise or imprecise version of multipleVersions (class TMTV1). At the end, the thread is suspended for a small interval and then it verifies if the deadline was missed (through variable dead). If the deadline was not missed the ScheduleManager is informed of the meeting of the deadline and information about this activation is stored in a object TaskActivation. During the execution of the thread, the JVM can detect the deadline miss, in this case it calls the class MissHandler, which is responsible for managing these events. Class MissHandler interrupts the execution of the task and informs the ScheduleManager of the deadline miss. The method waitForNextPeriod() is the last event, which informs to the $J V M$ about the ending of the period and prepares itself for the next activation. Figure 7 shows the code of the class ThreadMultVersions periodically executed.

The imprecise tasks implemented by objects multipleVersions are determined at the threads initialization in the class ExecutionTimeVariation as objects of class TMTV1. Class TaskMTV is abstract and serves as an interface for the imprecise tasks, as showed by figure 1 .

Class ScheduleManager plays a parallel role to the one of the class Scheduler. The objective of the ScheduleManager is to concentrate the process of adaptive scheduling, since that tasks call this class to determine which precision level should be used in their execution.

In order to guarantee mutual exclusion during the access to methods of the class ScheduleManager, they are implemented with the directive synchronized. It should be noted that some methods receive a RealtimeThread reference for the current thread as a parameter, since they need to have access to the imprecise threads, as in ((ThreadIC) th).setDead(false), whose function is to inform the task about a deadline miss. Figure 8 shows the code of ScheduleManager. 


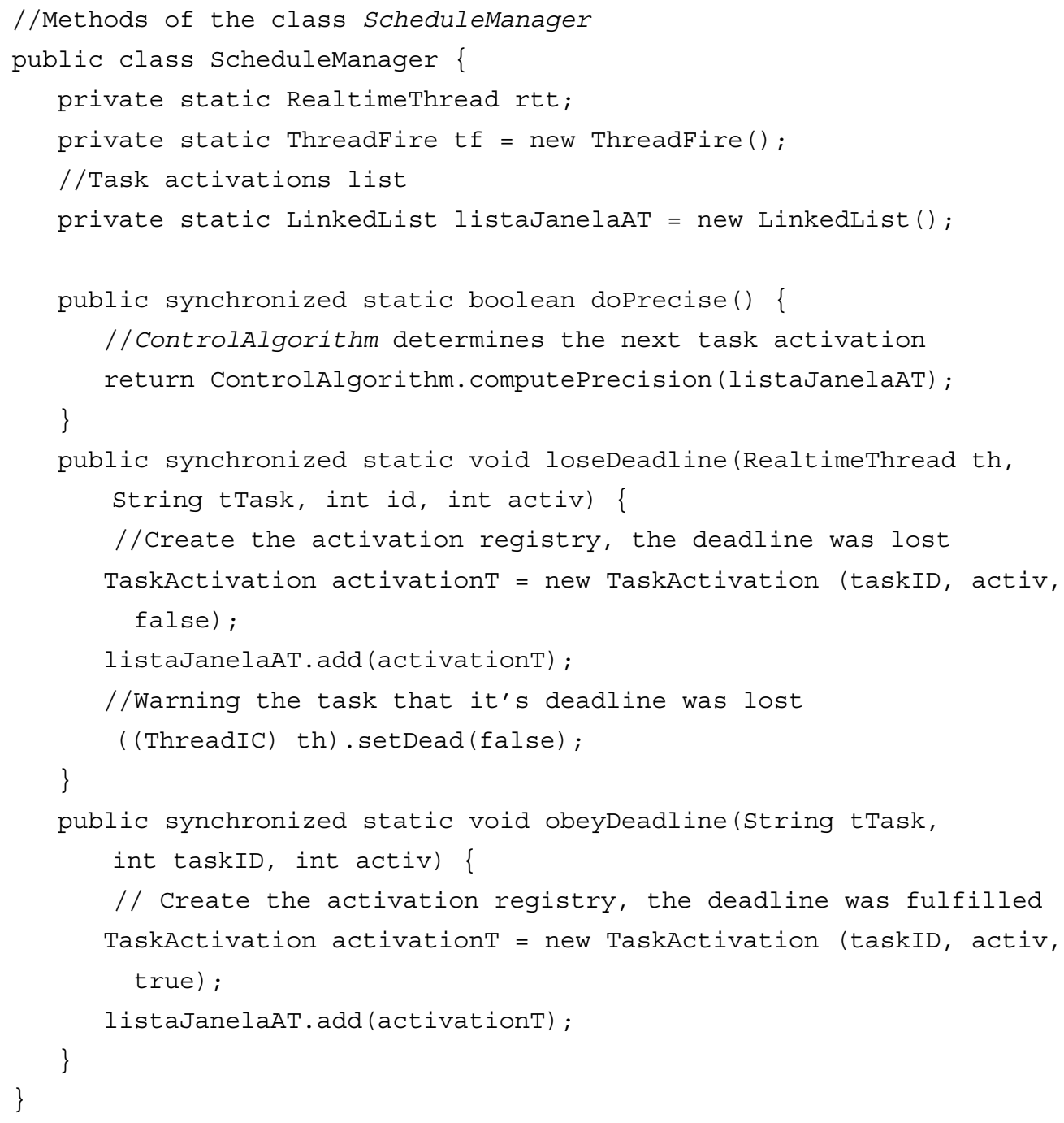

Figure 8. Part of the code for class ScheduleManager.

\section{Example of an adaptive scheduling algorithm}

Class ControlAlgorithm is responsible for deciding if the adaptation will be applied to the task. In the implementation of class ControlAlgorithm the adaptation algorithm used is very simple. Only the characteristics of the activations (object TaskActivation) were considered by the scheduling algorithm. More complex algorithms probably would use more information. For example, descriptors for the threads managed by the system with all the data of each task.
Objects of the class MissHandler are associated to imprecise threads in the constructor of ThreadIC. The purpose of these objects is to manage the deadline misses and to inform to the ScheduleManager so that the adaptation can be applied to the task. The constructor of MissHandler assigns the maximum priority to the MissHandler thread. The method handleAsyncEvent() is called by the $J V M$ when the deadline miss is detected and its function is to inform the ScheduleManager of the deadline miss (loseDeadline()). Then it reschedules the task again, with the method schedulePeriodic(). Figure 9 shows the implementation of class MissHandler. 


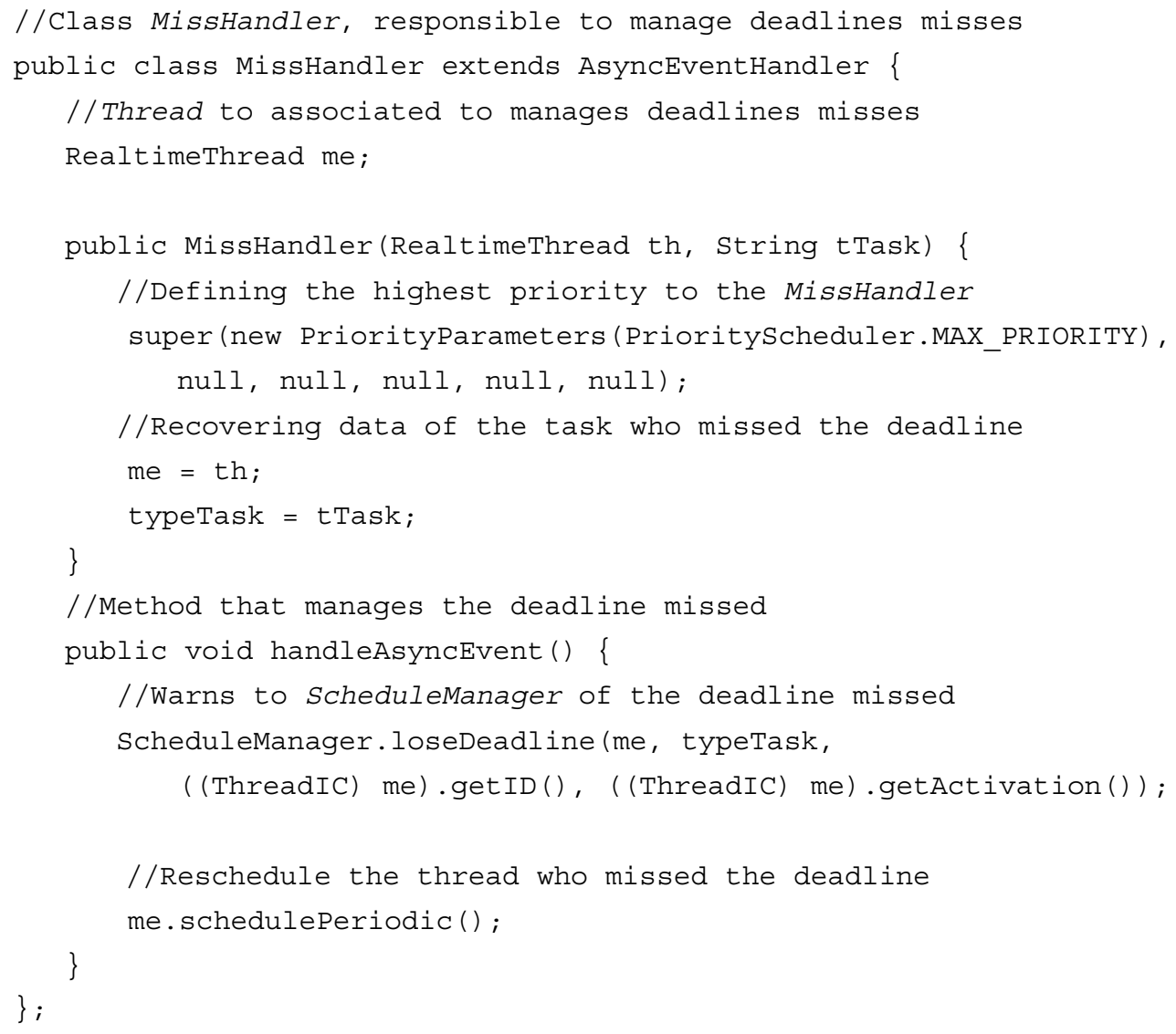

Figure 9. Part of the code of class MissHandler.

\section{Experiences}

As said previously, the implementation of the tasks with multiple versions was developed for periodic threads, since this facilitates the analysis of the adaptation of the imprecise tasks. The task parameters are defined during the initialization of the threads in class ExecutionTimeVariation. The most relevant parameters used in the experiences are the same for all the tasks: start at $1000 \mathrm{~ms}$, period of $2000 \mathrm{~ms}$, computation time of $2000 \mathrm{~ms}$, priority 10 and the specific task with multiple versions.

The computation done by the application tasks is only a simulation of some mathematical algorithm with the objective of consuming processor time until a deadline is either missed and, then, the task is submitted to a variation of its execution time. The computational cost of the task is increased at each period until, as consequence of a deadline miss, the adaptation calls the imprecise version of the task and its computational cost is reduced to avoid future deadline misses.

The adaptation algorithm in class ControlAlgorithm stores the states (deadline met or missed) of the last activations, defined by the variable activationWindow. The adaptation algorithm consults these activations to determine the precision level of the task to be executed next. If some activation within activationWindow lost its deadline, then the imprecise version of the task will be executed. The graphic in figure 10 shows the adaptation of a task implemented with multiple versions. 


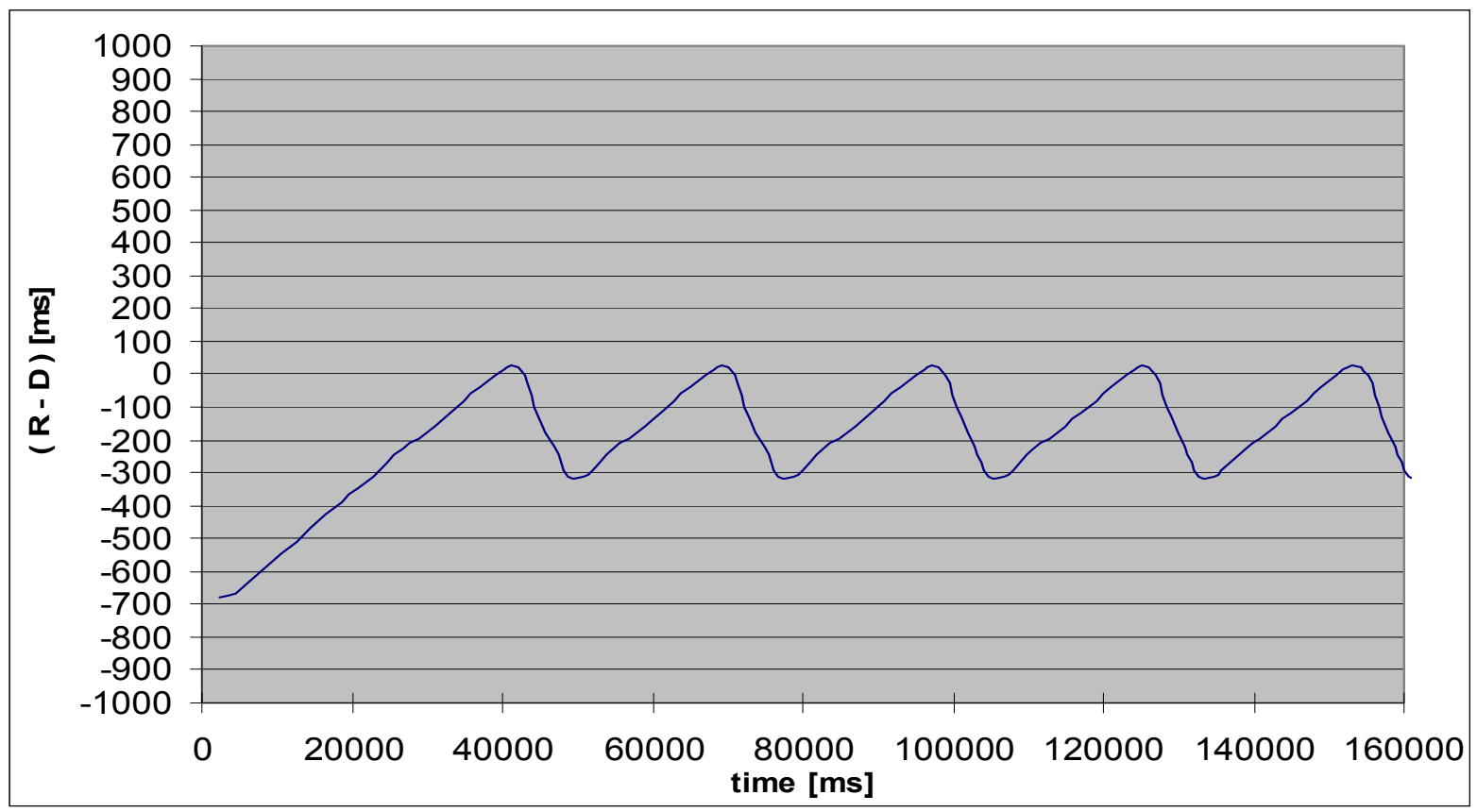

Figure 10. Example of an imprecise task with multiple versions.

The graphic shows the difference between the conclusion time and deadline of the task at the vertical axis, as a function of the time, which is at the horizontal axle. While this difference has a negative value it indicates that the task is meeting its deadline. But at the instant that this value becomes positive, it is necessary to adapt the execution time of the task so as it will meet the temporal requirement again.

A problem observed was that, as the application task does a mathematical calculation, the processor utilization by the task is high and constant. During some tests it was observed that by the ending of a period, in which the deadline was missed, the event created to signal this problem does not have a chance to be handled, because the next activation of the task starts its execution. This is a problem that the Java Virtual Machine used presents when facing asynchronous events (AsyncEventHandler) and priority inversion (the higher priority task does not interrupt the execution of the lower priority task). It was confirmed by Corsaro et. al. [9]. In order to give a chance for this event to be treated it was included a sleep() call with the objective to suspend the current thread.

\section{Conclusions}

The objective of the design pattern proposed in this paper was to facilitate the development of real-time tasks for adaptive scheduling based on the variation of the execution time.
In some situations it is not possible to use periodic real-time threads, implemented as RealtimeThread objects with the PeriodicParameter parameter, in the Java Virtual Machine of the Reference Implementation (JVM-RI [5]). Many of its methods are not implemented correctly (for example, the method responsible for the change of a task period). However, whenever it is possible to use this type of thread, the development of periodic real-time tasks becomes much more simple.

The design pattern described in this paper was defined and planned at the application level. The adaptation only actuates on the tasks created by the application itself. However, some classes of this proposal execute functions very related to the services of the operational system or of the $J V M$. For example, classes ScheduleManager and ControlAlgorithm, whose functions are similar to the $J V M$ scheduler. In future works new schedulers with adaptation based on imprecise tasks can be developed and be incorporated to the Java Virtual Machine.

Finally, it can be noticed that the use of the proposed class structure, as described by the diagram in figure 1, allows the development of tasks based on the multiple version technique and the objective of this work was accomplished. Analyzing the results it is observed that the tasks, executed periodically, are adapted always that the $J V M$ detects a deadline miss, deciding for the execution of the imprecise version of the tasks as a way to fulfill its temporal requirements. 


\section{References}

[1] G.Bollella, B.Brosgol, P.Dibble, et al. The Real-Time Specification for Java. Addison-Wesley, 2001.

[2] P.C.Dibble. Real-Time Java Platform Programming, Prentice-Hall, 2002.

[3] A. Wellings, Concurrent and Real-Time Programming in Java. Wiley, 2004.

[4] J. Rumbaugh, I. Jacobson, G. Booch, The Unified Modeling Language Reference Manual. $1^{\text {st }}$ Ed.. AddisonWesley, 1998.

[5] TimeSys Corporation (Embedded Linux \& Development Products): www.timesys.com. January, 2005.

[6] L. Chenyang, J.A. Stankovich, T.F. Abdelzaher et. al. Performance Specifications and Metrics for Adaptive RealTime Systems - RTSS. Florida, 2000.

[7] J.W.S. Liu, K.-J. Lin, W.-K. Shih, et. al. Algorithms for Scheduling Imprecise Computations. In: IEEE Computer Society Press, CA, 1991.

[8] J.W.S. Liu, K.-J. Lin, W.-K. Shih, et. al. Imprecise Computations. In: Proceedings of the IEEE, January, 1994.

[9] A. Corsaro, S. Douglas, The Design and Performance of Real-time Java Middleware. In: Transactions on Parallel and Distributed Systems, November, 2003. 